\title{
Integration of a Physical Model into the Realization of Engineering Changes in Manufacturing Systems
}

\author{
Daniel Cichos ${ }^{1, a^{*}}$ and Jan C. Aurich ${ }^{1, b}$ \\ ${ }^{1}$ Institute for Manufacturing Technology and Production Systems, University of Kaiserslautern, \\ Germany \\ adaniel.cichos@mv.uni-kl.de, bpublications@mv.uni-kl.de
}

Keywords: Planning; Manufacturing; Productivity; Simulation; Engineering Changes

\begin{abstract}
Manufacturing companies have to implement engineering changes in manufacturing systems to remain competitive in changing conditions. Physical influences like collisions or load capacities have to be considered for proper planning of these changes by modeling and simulating the influences. To consider these physical influences, a framework is presented for the integration of physical model into realization of engineering changes. The framework provides information for the feasibility of the work steps for the execution and enables an efficient planning. Hence, planning mistakes can be detected before changes are executed and the negative impact on ongoing manufacturing can be avoided.
\end{abstract}

\section{Introduction}

Due to changing demand and new technologies, manufacturing companies must constantly enhance their products, adjust their manufacturing program and at the same time reduce manufacturing costs. To adapt existing factories to these developments, a large number of engineering changes have to be carried out [1]. The prior planning of engineering changes in manufacturing systems is an important factor for the efficiency of the subsequent implementation [2]. During the planning process, a number of physical influences like collisions or load capacities have to be considered, as mistakes in planning lead to complications in execution [3]. In particular, unwanted physical influences lead to problems due to physical restrictions such as overall dimensions, weight or times. As a result of complex effects during the engineering changes, planning processes must be repeated and dates cannot be kept [4]. This requires a replanning and in turn causes high costs. To improve planning and avoid errors, a physical modeling is carried out during the planning of engineering changes in manufacturing systems. This physical modeling allows the simulation of the work steps, which are necessary for the execution of the engineering changes. Errors can thus be detected and corrected at the planning stage.

\section{Objective}

The objective of this paper is the integration of a physical model into the process for realization of engineering changes in manufacturing systems to simulate physical influences. In this paper, first, the state of the art is discussed and second, the procedure for taking into account the physical influences of engineering changes is described.

\section{State of the art}

Various concepts have been developed to improve the planning and execution of engineering changes in manufacturing systems. A standardized approach was developed for the processing of individual engineering changes in manufacturing systems [5]. The method handles the initialization, execution and follow-up of engineering changes. This takes into account the relationship of the affected manufacturing equipment, in particular based on the material flows. For this purpose, the work plans of the affected manufacturing equipment are used and the underlying material flows are compared. Another approach investigated the impact between manufacturing equipment [6]. A 
system was developed to analyze the impacts of engineering changes. The impacts on layout and process chains as well as the completeness and the conflict relationships with other resources were analyzed.

Based on the above-mentioned approaches, a concept for the planning and control of several engineering changes was developed [7]. The concept enables to merge engineering changes and implement them in parallel. It takes simultaneously into account the impact on the current manufacturing systems, the resources and the corresponding planning intervals. Nevertheless, this concept has weaknesses in the detailed planning of the work steps. Required work steps are only retrieved from an experience-based database. The required work steps are selected based on stored values. However, if only small deviations to the stored values in the database exist, they cannot be checked and errors can occur during planning. The selected work steps can lead to complications during execution due to deviations to the physical restrictions and cause considerable costs due to the necessary re-planning or manufacturing shutdowns.

In order to carry out a correct planning of the work steps, a physical model for a digital physical validation is necessary. A physical model maps all relevant physical sizes and enables the simulation of physical influences. So the required information for a digital physical validation can be provided. With an efficient change process using a physical model, the work steps can be planned with a high level of detail and the physical influences of the execution of the changes can be simulated. A concept for the implementation of a physical model for engineering changes already exists [8]. The physical influence of the work steps can be simulated via the physical modules of the model. However, this model needs to be integrated into the change process.

\section{Framework for engineering changes in manufacturing systems}

The change process of engineering changes in manufacturing systems is divided into three phases: preselection, planning and execution. Each of this Phases has different steps. Some of these steps modifies with the opportunities of the physical model. Figure 1 shows the connections between the physical model and the steps of the change process. The steps in which the physical influences are relevant are written in italics under each of these three phases in Figure 1. In this paper, a framework is developed to address all the elements of the three phases of the engineering change process described above. This new framework is described next. The focus is on activities, which can be supported with the physical model.

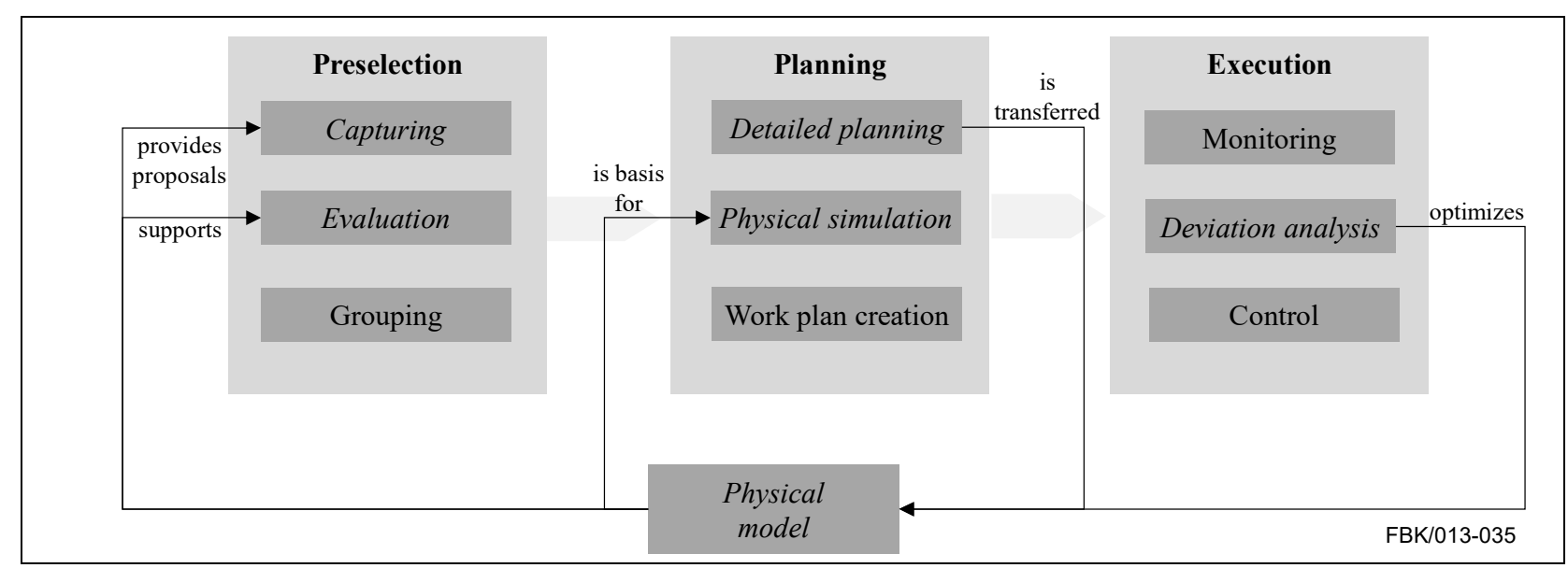

Figure 1: Connections between the physical model and the steps of the change process

Preselection. The procedure to realize engineering changes begins with the preselection. The flow chart is shown in Figure 2. At the beginning, there is a demand for engineering changes. This can be captured conventionally manually, for example through employee proposals or can be derived from the physical model. For the model-based capturing, the manufacturing system is displayed in a 3D 
environment. The manufacturing processes can be analyzed. Weakness can be identified by means of throughput times and costs of individual process chains. Further, other physical factors, such as the kinetics of the process steps, can be analyzed by physical modeling. In this way, for example, the largest cost drivers can be identified and replaced by more cost-effective solutions in order to save costs or inexpedient process sequences can be found and optimized. Based on the demand, the necessary data for the evaluation of the engineering change must be collected. Hereby, the physical model can support, too. The data is first imported from the physical model. If missing data is detected when the completeness is checked, this data is collected manually. If the data is complete, the evaluation follows. A qualitative evaluation for the selection of the engineering changes takes place. For the engineering changes, a value-of-benefit, economic and risk assessment is carried out. If the assessment is ambiguous, the physical model can complement the assessment by additional analyses. For example, different scenarios can be simulated in the current model. At the end, the changes are grouped and transferred to planning.

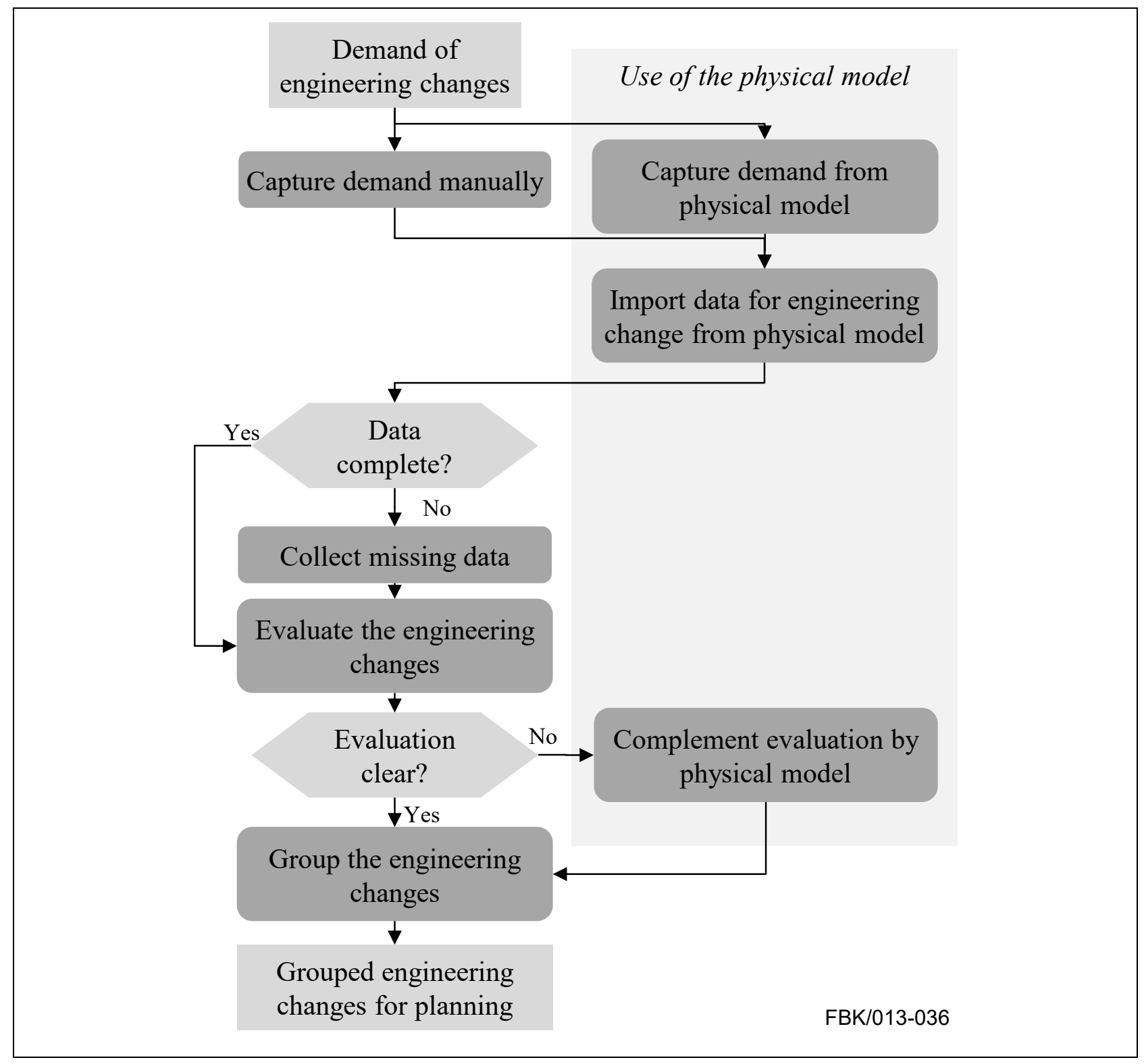

Figure 2: Procedure for the preselection

Planning. After the preselection, the planning of the changes starts. The flow chart is shown in Figure 3. At the beginning, there are the grouped engineering changes resulting from the preselection. Based on the conventional method, detailed planning is carried out first. Among other things, the changed layout of the manufacturing has to be created. In this case, usually several 
variants are created. The variants describe the manufacturing processes, the necessary equipment and layouts. The planning results are transferred to the physical model to simulate the changes. By comparison with the previous layout, the affected elements and areas of manufacturing are identified and this data is transferred to the physical model. By means of the simulations, errors or conflicts of the planning are checked. Based on the interconnections in the model, the effects and impacts of the respective engineering changes will be determined. If conflicts arise, detailed planning must be repeated and the planning results adjusted accordingly. If the planning is conflictfree, the work plan can be created. For this purpose, the necessary work steps are derived from the planning results. The work steps, in turn, are transferred to the physical model and simulated. If the simulation is not satisfactory or the simulation shows improvement potential, the work steps has to be adjusted. Otherwise, if no improvement potential is found, they can be scheduled chronologically and transferred to the execution.

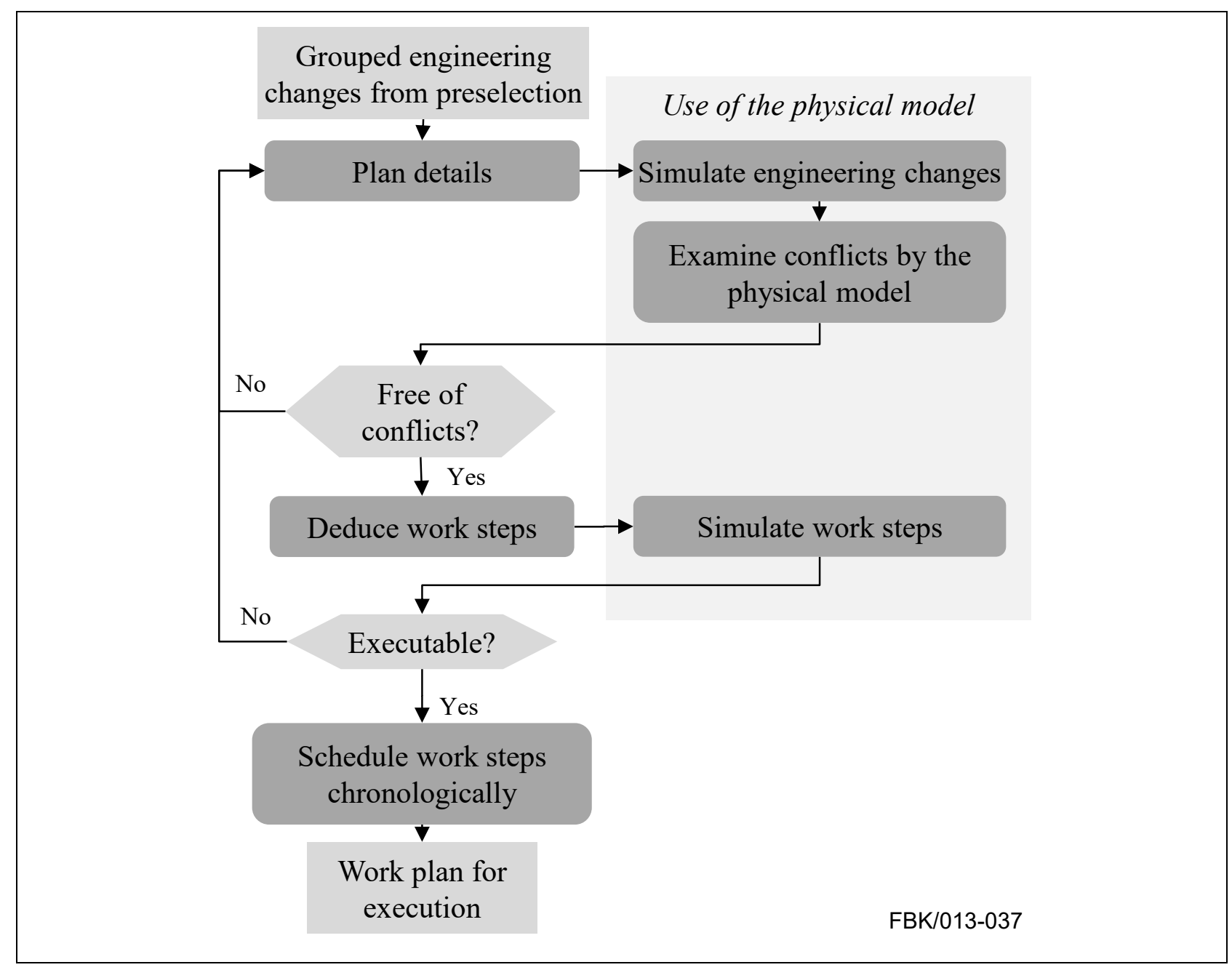

Figure 3: Process for the planning

Execution. The execution is carried out after the planning. The flow chart is shown in Figure 4. The starting point is the work plan of the planning. The work steps are executed and monitored. In this case, it is continuously checked whether there are any deviations between work plan and execution. If there are no deviations, the work plan can be carried out until it is finished. If deviations occur, a deviation analysis is carried out. Among other things, the root cause of the deviation is determined. Corresponding countermeasures are taken and the execution is continued. Further, it is examined if the deviations should have been indicated in the simulation of the changes in the physical model, which means that there is a discrepancy between the reality and the physical model. Often, unexpected events may delay execution and the schedule that was created cannot be met. If there is a possibility that the events occur more frequently, they must be taken into account in the physical 
model and the model must be optimized regarding this. If this is the case, the physical model has to be adapted accordingly. To this end, either certain parameters must be adapted or the simulation algorithms must be revised. If there is no fault in the physical model, the cause of the error is due to unpredictable events.

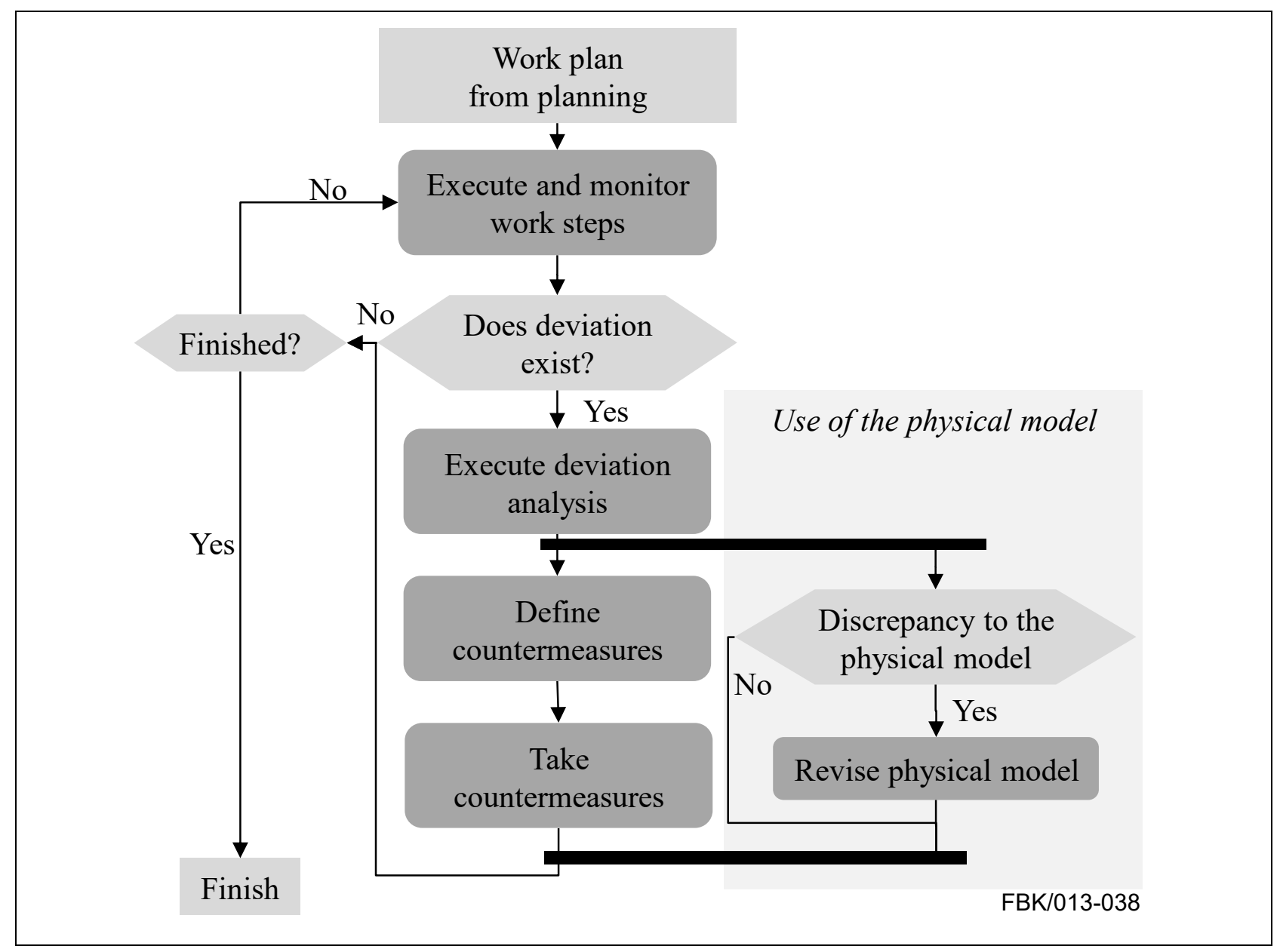

Figure 4: Process for the execution

\section{Validation}

For the validation of the framework, the manufacturing system of the turbine wheel for a turbo charger is used. The manufacturing system exists of a production line with 10 machines. Based on the physical model, a chart with the workload of the machines in the manufacturing system is created to capture existing change demand (Figure 5). The chart shows a full workload of the balancing machine and a very low workload of $30 \%$ and $50 \%$ of the washing machines. Because of the $100 \%$ workload of the balancing machine, a high buffer stock occurs in front of this machine. Based on this information, two engineering changes are defined. Engineering change 1 is to add an additional balancing machine. Engineering change 2 is to remove one washing machine. Because there are two washing machines with an average workload under $50 \%$, one machine can be removed. The evaluation of these two changes shows a clear result to realize these changes. The changes are grouped in one group, because both changes affect the same area and production line. 


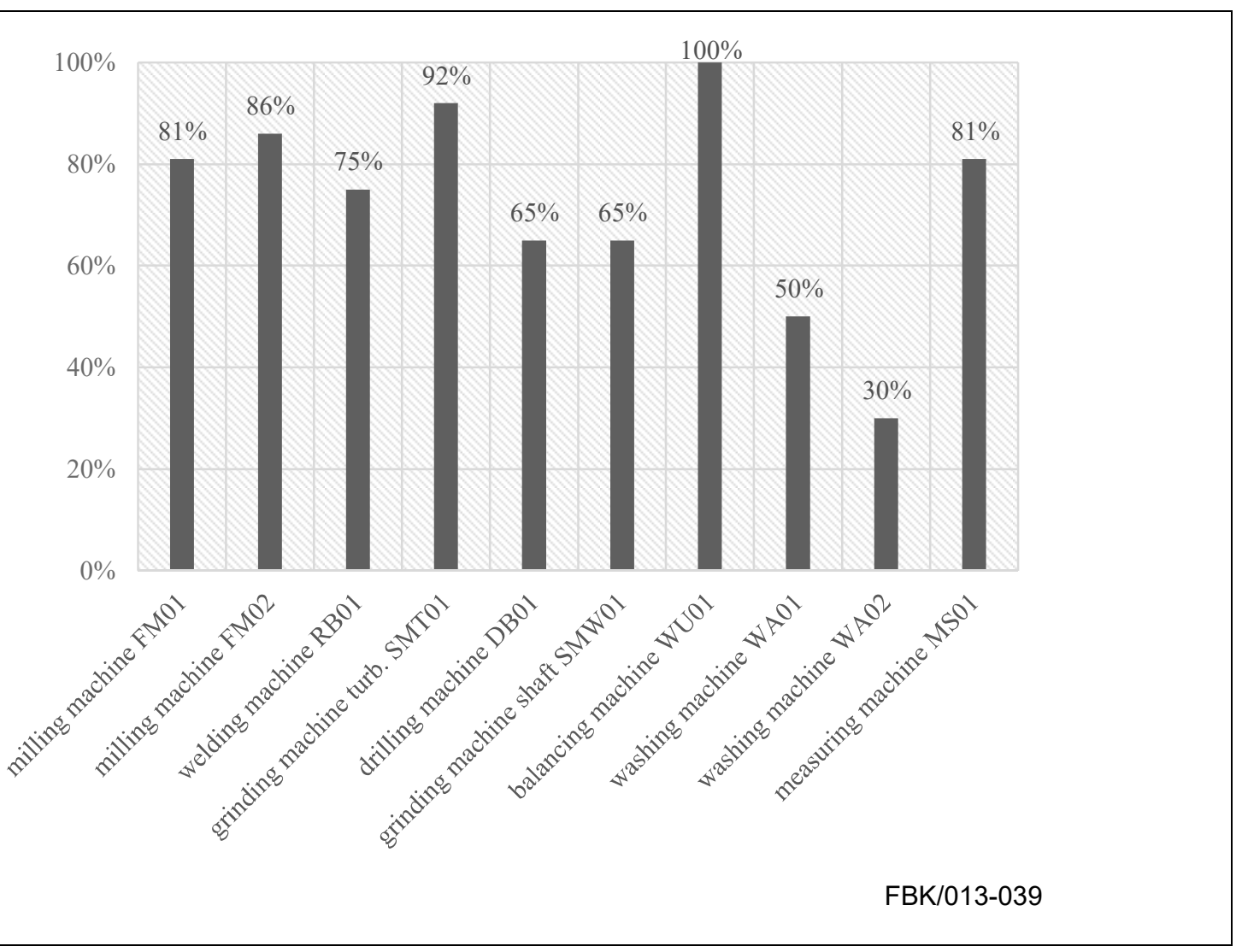

Figure 5: Chart with the workload of the machines

After the preselection, the new layout and manufacturing details are planned in the software demonstrator (Figure 6). During this planning, the simulations to examine conflicts of the changed manufacturing systems are carried out automatically in the physical model. The new process times are simulated and it shows a better workload of all machines, cause of a possible reduction of the cycle time. Based on the planning results, the work steps for the execution are deduced. The transportation work steps are modeled and simulated in the software demonstrator. Based on the transport path, a collision control is carried out. The first version of the work plan for the execution contains a collision while removing the washing machine. To avoid this collision, a part of the production line have to be disassembled. This work step is added to the work plan and the work steps are scheduled chronologically. The execution takes place according to the new version of the work plan. While monitoring the work steps, no deviations occur, which points out the functionality of the physical model. 


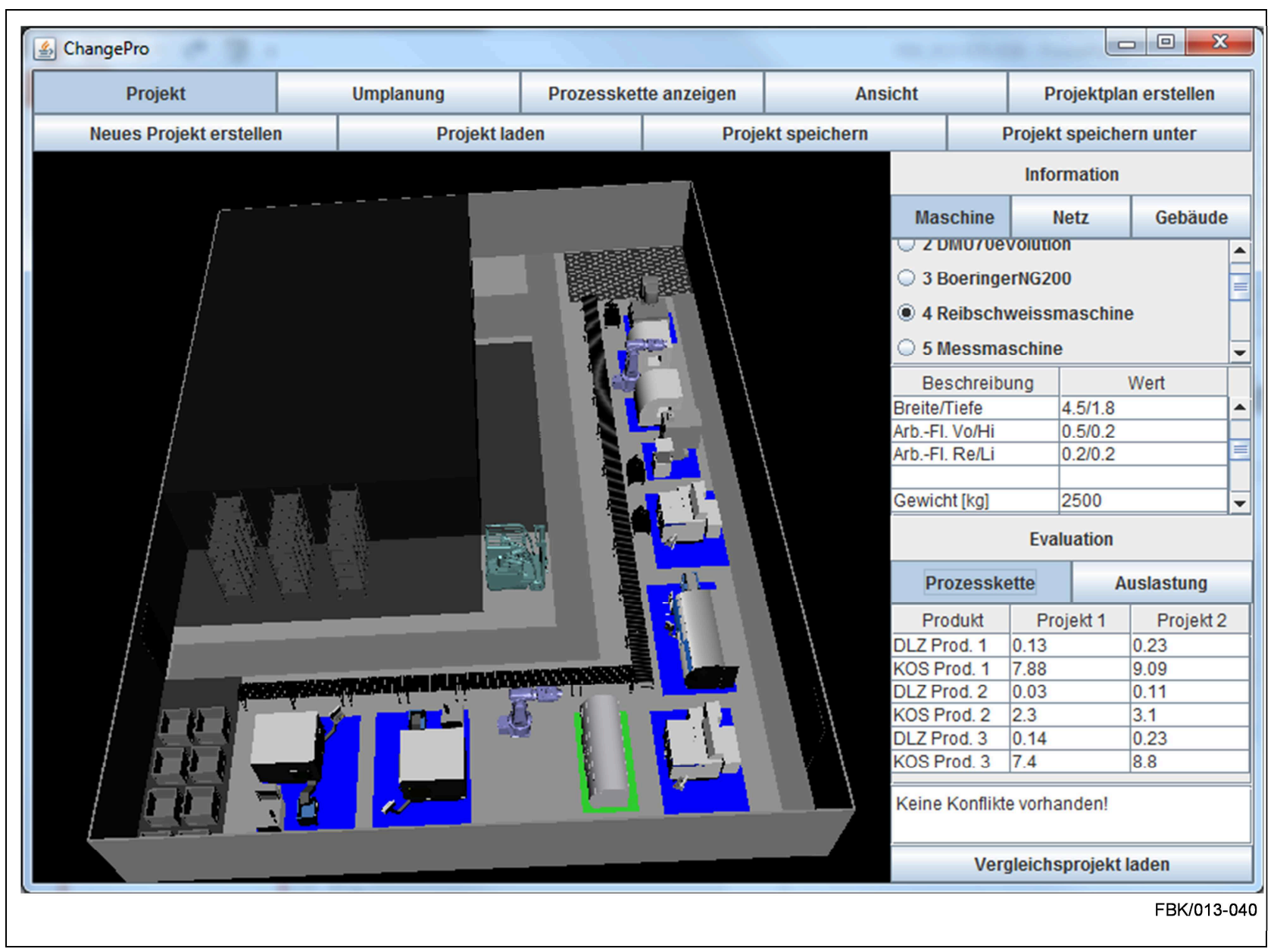

Figure 6: Planning results of the software demonstrator

\section{Conclusion and Outlook}

The presented framework allows the support of engineering changes by a physical model of the manufacturing system. Thereby, planning can be significantly improved, because errors can be detected at an early stage by simulating the work steps and can be prevented during planning.

In further work, the software demonstrator has to be extended. Therefore, the interfaces between the existing simulation methods and the physical model have to be defined. The data needed for the simulation in the physical model and the resulting data needed for the realization of the changes have to be determined. Moreover, the handling with this data has to be described.

\section{Acknowledgment}

The results of this article are taken from the project IRTG 2057 "Physical Modeling for Virtual Manufacturing Systems and Processes" funded by the German Research Foundation (DFG).

\section{References}

[1] F. Kühn, Digitale Fabrik - Fabriksimulation für Produktionsplaner, Hanser-Verlag, München, 2006.

[2] C. Kunz, Strategisches Multiprojektmanagement - Konzeption, Methoden und Strukturen, 2. Aufl., Dissertation, Universität Bamberg, Bamberg, 2007.

[3] J. Seidel, T. Ziegler, Management von Projektabhängigkeiten, in: C. Steinle, F. Ahlers, M. Barnert (Eds.), Handbuch Multiprojektmanagement und -controlling. Projekte erfolgreich strukturieren und steuern. 2., neu bearb. und erw. Aufl., Schmidt, Berlin, 2010, pp. 115-129. 
[4] M. F. Zaeh, L. Papadakis, M. Langhorst, Simulation of the manufacturing process chain of welded frame structures, Production Engineering 2 (2008) 4: pp. 385-393.

[5] M. Rößing, Technische Änderungen in der Produktion - Vorgehensweise zur systematischen Initialisierung, Durchführung und Nachbereitung, in: J. C. Aurich (Ed.): Produktionstechnische Berichte aus dem FBK, Bd. 02-07 (2007).

[6] R.C. Malak, Methode zur softwarebasierten Planung technischer Änderungen in der Produktion, in: J. C. Aurich (Ed.): Produktionstechnische Berichte aus dem FBK, Bd. 05-13 (2013).

[7] D. Cichos, J.C. Aurich, Planning and controlling of multiple, parallel engineering changes in manufacturing systems, Procedia CIRP 33 - Proceedings of the 9th CIRP Conference on Intelligent Computation in Manufacturing (2015) 81-86.

[8] D. Cichos, J.C. Aurich, Technische Änderungen in der Produktion - Implementierung eines physikalischen Modells, wt Werkstattstechnik online 9 (2016) 671-675. 